\title{
Towards a Technogenic Affordance
}

\author{
Stefan Palitov \\ Aalborg University \\ A.C.Meyers Vaenge \\ Copenhagen 2450, Denmark \\ stefan.palitov@gmail.com
}

\section{INTRODUCTION}

As the human practice becomes more technological, various cultural, economic and social interactions become dependent on what technologies afford us to do. There is an obvious possibility for this relationship to spread over large parts of the human experience. This can become a constantly present vail of interpretation between the individual and its perception of the world. As these technologies become more ubiquitous, human perception and ability to act will become dependent on technological, produced affordances. This eventually leads to a novel form of the negotiations for how the world should be seen and acted upon. Possessing the technologies that have affordances for such interactions will become critical in building up and maintaining the position of power. In its extreme, this struggle applies over the right to control the affordances for perception of reality and the very ability to act upon it in any meaningful way.

This demands clearer understanding of the properties of the affordances residing in a contemporary, highly technological eco-system that enables both access to information and agency to humans that are integrated with such systems. The goal is not to reinvent the affordance as such, but to locate it into the technological and more importantly, to understand the dynamics that promote it as a technogenic affordance. Additionally, with the spread of Extended Reality (XR) technologies the technogenic affordance opens epistemological issues that become unavoidable in the discussion.

This paper is built on two main concepts. The affordance is understood in the context of the materials that poses it. When these materials become part of a technology, they create new and designed affordances. Therefore, the affordance is tied into the technogenic relationship. As it will be addressed later in the paper, this human-technology coupling is understood in the context of Hayle's position on technogenesis with all the implications following such interactions.

\section{THE EMERGENCE OF THE TECHNOGENIC AFFORDANCE}

How do we engage with the technogenic affordance? The crucial difference between the affordance and its subset technogenic affordance is that the latter is produced. Both Norman (Norman, 2013), Gaver (Gaver, 1991) and to some extent Gibson (Gibson, 1979), have previously observed the possibility of the produced affordance. Where they note that some affordances can be produced, I note that the all of the technogenic affordances have to be so. Building on these positions, I propose to address the affordance in the feedback loop that emerges in the process of technogenesis. Additionally, my position draws on Gaver's idea of sequential affordances, a concept he introduces to address the situations when acting on some affordances gives information on new affordances.

The production of the technogenic affordance can arise from the activity of both humans and autonomous intelligences. Its effects may be of intended or unintended nature. The ability to shape affordances according to our needs and imagination makes them active participants in how the world is seen and acted upon, which in turn expands or limits our actions. This interaction demands responsibility for understanding the epistemological and behavioural changes that will emerge from such systems. The technogenic affordance is not simply encountered but manufactured and as such it can be changed for better or worse.

\section{THE CONCEPT OF THE AFFORDANCE}

The basic understanding of the affordance is given by James Gibson who positions it directly into the environment and ties it down to the physical properties of objects and spaces. He argues that the affordance is a combination of these physical properties that afford or enable the animal to take an action. "The affordance of anything is a specific combination of the properties of its substance and its surfaces taken in reference to an animal" 
(Gibson, 1979). The affordance does not exist outside the object, but it is not a mere property of it. It exists as a potential use of the property of the substance from which an object is built.

However, he accepts the influence of humans over the affordance due to scientific and technological progress. The shapes and substances with all of their properties are altered to increase the benefits available to the species (Gibson 1979). With that in mind, it is clear that the affordance is directly connected to the functionality of the object or the space that it resides into.

Allowing human agency to affect the state of the affordance, inevitably expands the possible locations and forms in which the affordance can exist. The affordance can now migrate from the properties of objects and environments in nature into human-made objects. By creating technologies and systems that allow us to perceive and act in novel ways into the world we build the affordance inside these technologies and systems. Now the affordance becomes a relationship with the tools of our making and through the proliferation of autonomously developing systems, with tools of their own making. The affordance doesn't simply occur in these technologies but it arises from mostly intentional design. Even more than before, the intentionality towards the technologically produced affordance becomes dominant way of thinking as part of its design process. This is pronounced in Don Norman's understanding of the affordance from the point of view of his design theory.

"The presence of an affordance is jointly determined by the qualities of the object and the abilities of the agent that is interacting. This relational definition of affordance gives considerable difficulty to many people. We are used to thinking that properties are associated with objects. But affordance is not a property. An affordance is a relationship. Whether an affordance exists depends upon the properties of both the object and the agent." (Norman, 2013).

The relational approach is more useful to us if we accept that we are making technologies that have affordances which are different from the affordances we find in nature. They may be designed to mimic or simulate physical affordances, but also to offer completely new bodily and cognitive functions. This means that we are in direct connection with the making of the affordance, in sharp contrast with our reactive relationship with affordances encountered in the nature. However, the produced affordances increase in complexity and may require extensive education to reveal their presence. Today we are surrounded with various systems that afford us to observe and to act into the world through methods that sometimes we do not grasp in their totality. This becomes a critical issue. The phenomenological school of thought demonstrates that the intention to act upon the surrounding is crucial to the embodiment and if we follow this stance completely, to consciousness and to reality.

\section{HUMAN-TECHNOLOGY SYMBIOSIS}

Embedding the affordance in the technological, it becomes part of the processes that accompany human-technology dynamics.

As Katherine Hayles's notes, the ever-increasing dependence and co-evolutionary relationship between human and technology-the technogenesis, has been going since the first tools were invented (Hayles, 2012). Aside from enabling certain actions, being affordances on their own, these tools also promoted increased cognitive activity. This in turn increased the complexity of the tools built, which then again promoted development of different cognitive faculties. This interaction creates a feedback loop that promotes increased tempo of evolution, both to humans and to technologies. In recent times, due to the dominantly technological aspect of developed societies this interaction has become much more complex and dynamic.

"Contemporary technogenesis is about
adaptation, the fit between organisms and their
environments, recognizing that both sides of the
engagement (humans and technologies) are
undergoing coordinated transformations."
(Hayles, 2012)

Producing the human epistemological processes, social interactions and economic activities in a way to be co-dependant with technology, promotes a structural coupling between the two. Such interactions also presuppose a co-dependency to the extent that any information gathering, cognitive faculty and agency cannot be conducted without the sensor capabilities, data visualization, interconnectivity and affordances for agency of the technological systems. In the past the ideas for that structural coupling have been explored in various interactive media artworks to the extent that they demonstrate their inherently cybernetic nature (Ascott, 2003). Today we are seeing a tendency for this coupling to become unavoidable modus operandi in a contemporary environment.

The idea of merging of two systems is present in evolution theories such as the one of symbiogenesis. Symbiogenesis is the merging of two distinct organisms to form a single organism (Margulis, 1998). Some authors take the idea and place it the human-machine context. They see the same principles of human-computer coupling as being of symbiogenic nature which is defined by cooperation between the two organisms, the technological and the human. (Castellanos \& 
Gromala, 2009). Dispersing the notion of intelligence away from the anthropocentric view, we observe cognitive systems of various biological, mechanical and digital nature pursuing their own goals. The possibility for any degree of Al cognitive autonomy will make this symbiogenic nature even more prominent. Not venturing too far into contingencies, we can observe our behavioural changes when interacting with technology in our everyday life as part of this symbiogenic process. The technology requires inputs through our behaviour or intentions in a certain predesigned way in order to afford us agency for example, in the cyberspace. This merging must not be seen only as merging between the physicality of the human and the technology, but also other instances of the 'soft' parts of human existence, most notably social dynamics and information distribution. It is in this context in which the human-technology symbiogenesis becomes technogenesis. This symbiogenesis promotes a merging between technologies with their affordances and their producers. The affordance is not only encountered in nature but entangled in the intended and nonintended designs we and other autonomous intelligences produce.

Lack of awareness of this mutual influence, significantly impairs our ability to act in a changing eco-system of social, cultural and economic relations that are enabled by a complex system of technogenic affordances. There is a chance to lose the power of establishing facts and their interpretation into truth, to systems which are outside of our comprehension or possession. It is becoming increasingly difficult to understand this complexity of affordances that interact between themselves to create habitual niches.

"A niche is a set of affordances that enables certain modes of existence in the framing of the habitat." (Gibson, 1979)

The best example would be the internet and the interconnected devises that give us access to information and to the ability to act over the network. We can analyse the internet as a general affordance to access certain knowledge as well as an affordance to act. Then we have the entire machinery that affords the internet, with their own systems of sub-affordances. Algorithms and AI have the role of curators. They become affordances through which we are exposed to preselected content. The devices through which we access the network possess the affordance of connectivity. In a way, these devices afford us to get to new affordances. The act of searching the internet is to interact with a complex network of technology residing affordances, which in some instances afford each other. This creates the 'niches' in the same way Gibson saw them emerging in nature. Such niches are usually built on post-phenomenological relationships. Phenomena that are not otherwise accessible to technological sensors and human senses are translated into a language that the recipient understands (Idhe, 2009). This for example can include big data visualization which translates information into comprehensive human stimuli or EEG patterns used as inputs in various machinery.

\section{THE MONOPOLY OVER REALITY}

MEDIATIVE, a consumer behaviour research group conducts data analysis for many big companies, including Google. They are looking at how the design evolution of Google's search engine result pages (SERP) affects human behaviour. Using eyetracking technology, they produce heatmaps of where and how long the users look and which listings are clicked the most (MEDIATIVE, 2014). This data is valuable for Google, because the position of the most clicked listing has a greater value than the rest. It also demonstrates that the casual user does not spend much time and effort to do extended search on the list. The search pattern also slightly varies depending on the SERP design. However, the top entries are dominant and are looked at and clicked the most. In the basic, organic listing of results, 83 per cent of the people looked at the top entry and 34 per cent people clicked it. 76 per cent of the clicks were on the top 4 listings. Although the heatmaps of others SERP designs demonstrate distribution of attention to other elements, the users usually maintain the interest above the fourth entry that is given to them. This research was done for other purposes than observing the behaviour during a thorough research on a topic, but it may serve as a general pattern of behaviour in everyday search activities. This covers anything from product placement to news delivery. It also demonstrates that the control over the affordance to access information is curated by the several links that occupy the dominant places in the listings. Everything else, intentionally or not, is buried in the information noise.

Our behaviour with the SERP shows limited engagement with the information that is available to us. The example of the affordances of the internet and the distributed power over the access to information is only one way of controlling the affordance through design. In order to make this issue more visible I imagine a possible future in which extended reality (XR) technologies are widely adopted. Here we can directly observe the epistemological issues arising from the structural coupling and the technogenic affordances. Their effect becomes immediate when they directly and obviously alter our senses and thought processes.

I understand $X R$ as a state of a technologically mediated, first-person embodiment into an 
interactive environment. The spectrum goes from virtual reality (VR), which shuts us from the immediate environment transporting us to a world of our making, to augmented reality (AR) which superimposes stimuli and affordances for interaction over what we consider our objective reality.

The VR experience is predicated by the user's agreement that they trade their bodily stimuli and natural affordances to the reinterpretation done by the creators of the experience. However, with AR and the most likely continuation of the structural coupling between humans and technologies, the technogenic affordances of the system become integrated with the affordances of the surrounding world possibly altering the way we engage with the latter. This promotes outsourcing of the ability and eventually of the right to learn and interact with the world without the mediation of the machine. Although the current generation of technology favours the visual stimuli (Hansen, 2004), this does not limit the possibility to promote the relationship between other senses and technogenic affordances. Additionally, implants that alter cognitive functions afford specific way of processing the information that we perceive, adding an extra layer of technological interpretation. The reality then becomes whatever the technogenic affordance allows us to perceive and to do. This is possibly an inherent problem of using technology as a mediator or objective verification of the fact, instead of being understood as an epistemological, technogenic affordance of our making, biases and possible misuse. With the proliferation of $\mathrm{Al}$, that aspect may also expand. As the eye-tracking heatmaps have shown us, the truth is outsourced to the few listings of a Google search. Numerous ethical, legal and primarily philosophical questions arise from these relations. What happens when the capacity to interpret what we see, hear, remember or think is outsourced to technology? What happens when there is an additional unavoidable layer of interpretation over our experience of the world? To what extent will be the reality distributed to a $3^{\text {rd }}$ party gospel?

Critical for our ability to alter it is the awareness of the dynamics that define it. Here I propose an open list of properties of the technogenic affordance.

Technologically manufactured. It is not found in the physicality of the nature, but in the interactions with and through technological systems.

Conceived. Human and other intelligences may demonstrate intentionality towards producing affordances for self-imposed means.

Ubiquitous. It is becoming increasingly unavoidable part of our experience of the world. With the increase in structural coupling between humans and technologies this property may become dominant.
Niche. More often than not it is an affordance made out of affordances. It is part of a complex network of interdependencies between other affordances.

Hidden. Since it is part of a niche, its presence is not always evident. It is part of an opaque technological systems that require understanding of their complexities in order to get revealed.

Translatable. It demonstrates postphenomenological qualities to the extent that the perception and agency is partially or completely tied to the apparatus itself. Input-output connections may be created with arbitrary representations from one phenomena into another.

Data driven: The information generated from any measurable behaviour or occurrence can become an affordance for an output.

\section{REFERENCES}

Ascott, R. (2003) Telematic Embrace:Visionary Theories of Art, Technology And Consciousness, London: University of California Press Ltd.

Castellanos, C and Gromala, D. (2009). Symbiogenic Experiences in the Interactive Arts. In: After Media: Embodiment and Context. Proceedings of DAC 2009. University of California, Irvine.

Gaver, W. (1991) Technology Affordances. In: Proceedings of the SIGCHI Conference on Human Factors in Computing Systems. ACM, New York.

Gibson, J. (1979) The Theory of Affordances. In: The Ecological Approach to Visual Perception. Boston: Houghton Miffin.

Hansen, M. (2004) New Philosophy of New Media, Massachusetts: MIT Press.

Hayles, K. (2012) How We Think: Digital Media and Contemporary Technogenesis. Chicago: University of Chicago Press.

Ihde, D. (2009) Postphenomenology and Technoscience: The Peking University Lectures, NY: State University of New York Press, 2009.

Margulis, L. (1998) Symbiotic Planet: A New Look at Evolution, New York: Basic Books.

MEDIATIVE (2014) The Evolution of Google Search Results Pages \& Their Effect On User Behavior. http://www.mediative.com/whitepaper-theevolution-of-googles-search-results-pages -effectson-user-behaviour (2018)

Norman, D. (2013) The Design Of Everyday Thing, Basic Books. 